\title{
Developing a Highly Specific Biomarker for Germ Cell Malignancies: Plasma miR371 Expression Across the Germ Cell Malignancy Spectrum
}

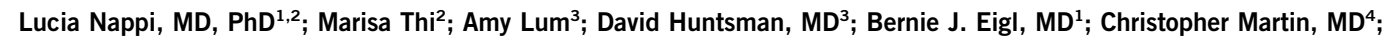 \\ Brock O'Neil, MD; Benjamin L. Maughan, MD, PharmD; Kim Chi, MD; Alan So, MD²; Peter C. Black, MD²; Martin Gleave, MD²; \\ Alex W. Wyatt, $\mathrm{PhD}^{2}$; Jean Michel Lavoie, MD, CM, MEng ${ }^{1}$; Daniel Khalaf, MD ${ }^{1}$; Robert Bell, MSc ${ }^{2}$; Siamak Daneshmand, MD; \\ Robert J. Hamilton, MD, MPH ${ }^{6}$; Ricardo R.N. Leao, MD, PhD ${ }^{6,9}$; Craig Nichols, MD ${ }^{7,8}$; and Christian Kollmannsberger, MD ${ }^{1}$
}

PURPOSE Our objective was to evaluate operating characteristics, particularly specificity and positive predictive value (PPV), by mapping plasma miR371 expression to actual clinical events in patients with a history of germ cell tumor.

PATIENTS AND METHODS One hundred eleven male patients with a history of or newly diagnosed germ cell tumors were evaluable. Biospecimens obtained before confirmed clinical events were analyzed for miR371 expression with blinding of providers and laboratory personnel to analytic results or clinical status, respectively. Cases (patients with clinically confirmed active germ cell malignancy [aGCM]) and controls (patients with no clinically confirmed aGCM) were assigned over the course of the management. Patients were assigned risk status (high, low, or moderate) based on the composite clinical picture at time points in management.

RESULTS Considering all cases and controls and results of prospectively obtained biosamples analyzed for miR371 expression, 46 (35\%) of 132 samples had clinically confirmed aGCM over the course of management; 44 (96\%) of these 46 patients had plasma miR371 expression (true positives) with no false positives. Two (4\%) of 46 patients had no miRNA expression despite pathologic confirmation of aGCM (false negatives). Plasma miR371 expression in confirmed aGCM had a specificity, sensitivity, positive predictive value, and negative predictive value of $100 \%, 96 \%, 100 \%$, and $98 \%$, respectively. Interpretation of sensitivity and negative predictive value is limited by modest follow-up. Specificity and sensitivity were $100 \%$ and $98 \%, 100 \%$ and $92 \%$, and $100 \%$ and $97 \%$ in the low-, moderate-, and high-risk groups, respectively, with a median follow-up time of 15 months.

CONCLUSION Plasma miR371 expression predicts aGCM with high specificity and positive predictive value. Although other operating characteristics of miR371 await longer follow-up for more complete definition, the findings of a highly specific liquid biopsy strongly support moving forward with large-scale, real-world clinical trials to further define full operating characteristics and to identify clinical utility and areas of patient benefit.

$\mathrm{J}$ Clin Oncol 37:3090-3098. @ 2019 by American Society of Clinical Oncology

ASSOCIATED CONTENT

See accompanying Editorial on page 3063 Data Supplement Author affiliations and support information (if applicable) appear at the end of this article.

Accepted on August 23, 2019 and published at jco.org on September 25, 2019: DOI https://doi. org/10.1200/JCO.18. 02057

\section{INTRODUCTION}

During the past 45 years, therapeutic management of germ cell tumors (GCTs) has progressively improved to the point that now we can anticipate that more than $95 \%$ of patients presenting with a newly diagnosed GCT will ultimately be cured and most will have a high quality of life with a near normal life expectancy. ${ }^{1}$ There remain unmet needs in terms of reduction of late effects and patient burden, cancer care delivery, and access improvement.

Largely in the realm of developmental biology and pathology, certain families of microRNAs have been discovered that are nearly invariably present in tumor tissue from patients with active germ cell malignancy (aGCM). ${ }^{2-5}$ Importantly, a number of investigators have found that aGCM either leaks or releases a specific
microRNA, miR-371a-3p (miR371), that can be reliably detected in serum and plasma. ${ }^{6-9}$ miR371 is involved in regulation of embryonic stem-cell differentiation and is the leading candidate for development as a liquid biopsy, high-fidelity biomarker. ${ }^{2,10} \mathrm{~A}$ number of retrospective studies suggest that serum miR371 expression may have some characteristics of an ideal biomarker. ${ }^{6,7,9}$ High specificity has been consistently reported in early studies. ${ }^{8,11}$ miR371 is stable in serum and plasma by virtue of its secretion in a protein-microRNA complex and is stable for years in frozen samples. ${ }^{12}$ Serum, plasma, or tissue miR371 expression is seen in nearly all patients with intact primary GCTs. ${ }^{6,9,13,14}$ Expression disappears rapidly in most patients with clinical stage I disease after removal of the primary tumor ${ }^{13,15}$ and persists or increases in patients with known untreated metastatic disease. ${ }^{9,14}$

\section{ASCO}


This elegant work has been largely unadvertised in clinical realms but recently caught the attention of the clinical academic GCT community. This community began to understand the potential clinical utility of a very specific, easily measured circulating biomarker and to design clinical trials that map behavior of these biomarkers during management of GCTs (ClinicalTrials.gov identifier: NCT03067181). These trials, in aggregate, should clarify the role of the lead candidate biomarker, miR371; provide biorepositories with expert annotation for further development of new circulating biomarkers as clinically useful; and improve care delivery in GCTs. Lead pilot trials have recently been completed or are nearing completion in North America and Germany and through the Swedish/Norwegian GCT Consortium.

We report a limited institutional North American study mapping plasma miR371 expression to actual clinical events in patients with GCT. The primary objective was to define the rate of true positives (TPS) and false positives (FPs) and the positive predictive value (PPV) of miR371 expression in patients with GCT at variable risk for aGCM. The intent was to provide data and practical experience to inform the design and development of large, real-world definitive studies with a goal to fully develop miR371 expression as a possible reliable liquid biopsy for patients with aGCM.

\section{PATIENTS AND METHODS}

\section{Trial Design and Iterative Adjustments}

Our trial was designed with several assumptions, which are outlined in detail in the Data Supplement. This pilot trial was never intended to be definitive but could provide valuable information and experience to inform the design and development of a definitive, prospective, real-world trial. A fitfor-purpose design using prospective collection with blinded and retrospective analysis of plasma miR371 expression after expert validation of clinical events of interest was used. This prospective-retrospective design removed the possibilities of biased interpretation of results and the potential influence of overall results by patient selection. Of note, this trial design using only patients with GCT and prospective collection of an integrated biomarker with retrospective laboratory analysis is the design used by all of the large ongoing trials.

Early results of non-time-dependent end points (miR371 TPs in patients with clinically confirmed aGCM and no FPs) and, serendipitously, the discordant results (miR371 true negatives [TNs]) in patients who were presumed on clinical grounds to have aGCM (Fig 1) were pivotal in terms of heightening focus, accelerated our development of large definitive clinical translational studies, and prompted additional collaborative efforts with other major GCT centers.

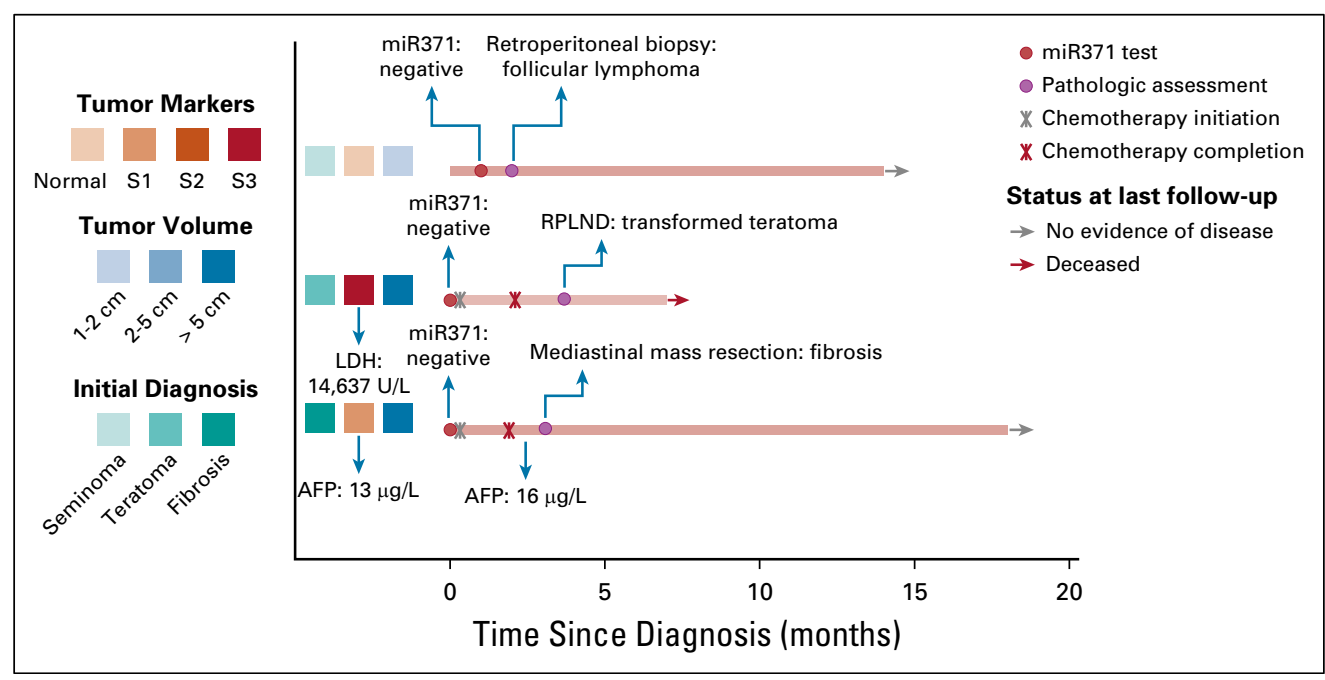

FIG 1. Clinical details of patients with true-negative microRNA results. Three patients presented with obvious clinical signs of germ cell tumor but no clinical confirmation of active germ cell malignancy (aGCM) and true-negative miR371 results. The first patient (from the top) was older, had pathologically confirmed testicular seminoma, and developed enlarged retroperitoneal lymph nodes (stage IIA) with negative tumor markers and confirmed growing pattern of the tumor by two sequential computed tomography scans. miR371 was checked after orchiectomy and was negative. A biopsy of the retroperitoneal lymph nodes revealed the presence of a follicular lymphoma. The second patient was diagnosed with extensive metastatic germ cell tumor. The pathology of the primary testis cancer showed a transformed teratoma; lactate dehydrogenase (LDH) was the only elevated tumor marker, and the miR371 was negative. After the chemotherapy, the residual tumor was resected, and the pathology showed only transformed teratoma. The third patient had a large mediastinal mass. A biopsy showed fibrosis, and he had low positive $\alpha$-fetoprotein (AFP; $13 \mu \mathrm{g} / \mathrm{L}$ ). miR371 was negative. After chemotherapy, his AFP was stably low (16 $\mu \mathrm{g} / \mathrm{L}$ ), and the pathology of the residual mass showed fibrosis only. RPLND, retroperitoneal lymph node dissection. 
With early evidence of uniform TPs and the lessons learned from three TNs, we realized there could be major practicechanging potential and favorable patient impact of miR371 expression analysis even if the only outstanding operating characteristic was high specificity and PPV for aGCM. Our pilot trial was refocused on important clinical scenarios with variable risk of aGCM in which a highly specific test would have maximum clinical utility and reduce uncertainty (eg, stage IIA disease, clinical stage I postorchiectomy and postchemotherapy residual radiographic abnormalities). In parallel, using the strong, early clues from our exploratory trial, we began to design and develop a definitive large realworld translational trial. SWOG concept S1823 was approved by the National Cancer Institute Division of Cancer Prevention in March 2019.

\section{Eligibility Criteria, Recruitment, and Data and Specimen Flow}

Our study was approved by the British Columbia Cancer (BCC) Ethics Board (H16-00934; date of approval: June 16, 2016). Eligibility included a new diagnosis or history of GCT. Patients were primarily identified through the Multidisciplinary Testicular Cancer Program at BCC, but later recruitment included BCC satellite clinics and the Huntsman Cancer Institute (Salt Lake City, UT) through approval by Huntsman Cancer Institute Institutional Review Board and material transfer agreements. Although generally consecutive patient enrollment occurred at BCC, it is likely that satellite clinic contributions, although still prospective, were not consecutive (Fig 2).

\section{Specimen Characterization Collection and Processing and miR371 Extraction, Analysis, and Assay Validation}

Blood samples were drawn in Cell-Free DNA BCT tubes (Streck, La Vista, NE) and processed and frozen as plasma aliquots, followed by analysis using microRNA extraction and analysis methods, as previously reported. ${ }^{11}$ Details of collection, processing, microRNA extraction, and analysis are available in the Data Supplement. miR371 expression was first quantified and reported as relative increase in the expression compared with negative controls by using the $\Delta \Delta$ Ct method. Results were classified as either detectable miR371 expression or no expression. Cut point determination is outlined in the Data Supplement.

\section{Statistics Analysis}

The receiver operating characteristic curves with the definition of the corresponding area under the curve, sensitivity, specificity, PPV, and negative predictive value (NPV) were established for miR371 and compared with the same parameters for computed tomography (CT) scans, $\beta$-human chorionic gonadotropin ( $\beta$-HCG), $\alpha$-fetoprotein (AFP), and lactate dehydrogenase ( $\mathrm{LDH})$. The repeatability and reproducibility of the results were evaluated by correlation coefficient and Pearson analyses. The data were analyzed using GraphPad Prism 6 (GraphPad Software, San Diego, CA).

\section{RESULTS}

\section{Patient Characteristics}

Overall, 132 samples from 111 male patients with a diagnosis of seminoma or nonseminoma were analyzed. One patient was partially unevaluable because of a missing postchemotherapy, pre-retroperitoneal lymph node dissection sample (Fig 2). Twenty-one patients contributed to the specimen risk designation with two samples collected at two different clinically relevant time points (before and after orchiectomy, $\mathrm{n}=3$; or before and after chemotherapy, $n=18$ ) and, therefore, were independently considered for the final analysis (Data Supplement).

\section{Operating Characteristics of miR371 in Cases With a History of GCT and Subsequent Confirmed aGCM and in Controls With History of GCT and No Evidence of aGCM Over the Course of Management}

We used a case-control construct, where cases were patients with a history of GCT who subsequently developed clinically confirmed aGCM and had a prospective biosample obtained in proximity to clinical confirmation and controls were patients with a history of GCT who had a prospective biosample available but never developed a relapse with aGCM. Controls could only be designated retrospectively after sufficient time had passed after biosample collection.

Individual swim lane plots visualize the time course of sample acquisition, clinical events, and coding of cases versus controls, and patients were clustered into low-, moderate-, or high-risk groups for harboring aGCM (Data Supplement). High-level characteristics (tumor size, pathology, stage, and marker elevation grouped by risk clusters) of all enrolled patient reflect a general GCT population (Data Supplement).

The case-control construct across 132 risk designations relative to miR371 expression with the operating characteristics calculated to date (June 15, 2019) is provided in Table 1. Median follow-up times for the low- and moderaterisk groups were 14.5 months (range, 4 to 35 months) and 15 months (range, 5 to 25 months), respectively. Across 111 evaluable patients enrolled and 132 evaluable risk designation settings, 46 (35\%) of 132 had clinically confirmed aGCM (gold standard confirmation by progressive imaging changes, pathology, and/or definitive or increasing classic marker elevation). Forty-four (96\%) of 46 patients with confirmed aGCM had miR371 expression demonstrated in prospectively obtained blood samples (44 TPs; no FPs; specificity, 100\%). Two of the 46 patients had confirmed (AFP $>400 \mu \mathrm{g} / \mathrm{L}$ and increasing, $n=1)$ or pathologically proven $(n=1)$ aGCM (Data Supplement) but had miR371 expression in the range defined as no expression (two FNs; sensitivity, 96\%; NPV, 98\%). The expression of miR371 in the case and control groups using a qualitative or quantitative relative expression of miR371 is shown in Figures $3 \mathrm{~A}$ and $3 \mathrm{~B}$, respectively.

Validation of reproducibility and repeatability of miR371 analysis was also performed. High concordance between 


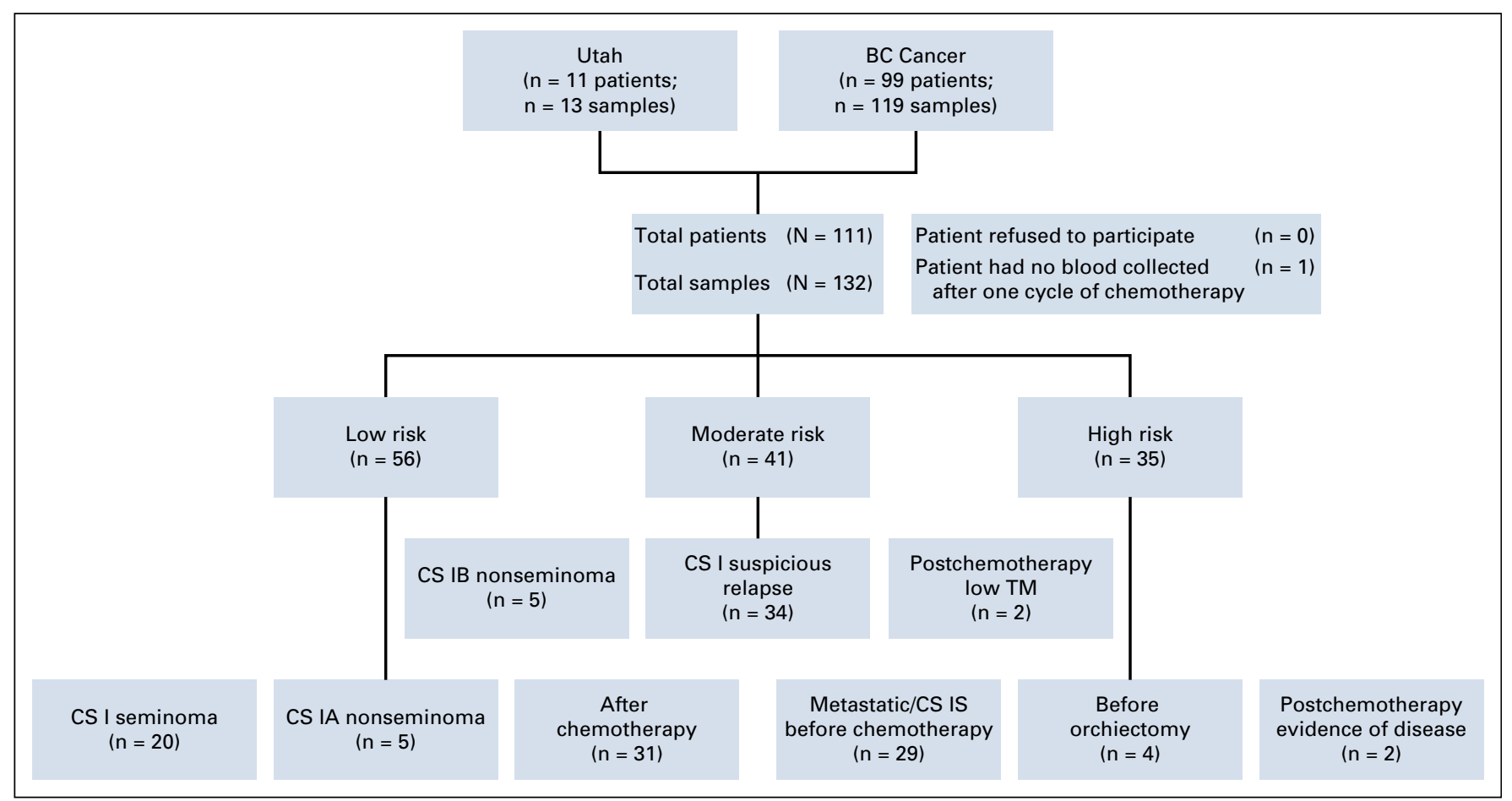

FIG 2. Schema of the study. Patients with germ cell malignancy from BC Cancer in Vancouver and the Huntsman Cancer Institute in Salt Lake City, Utah, were enrolled in the study. One patient had blood collected for miR371 before starting chemotherapy but not before surgery for residual viable disease and was considered unevaluable. The patient samples were divided into the following three risk categories, according to the probability of harboring active germ cell malignancy (aGCM): low risk ( $5 \%$ to $25 \%$ risk), which included patients with postorchiectomy clinical stage (CS) I seminoma and CS IA nonseminoma with no suspicious signs of relapse on surveillance and patients with or without residual radiologic disease after chemotherapy and normal tumor markers (TMs); moderate risk (25\% to 50\% risk), which included patients with postorchiectomy CS IB nonseminoma with no suspicious finding of relapse, patients with CS I seminoma and nonseminoma with clinical signs of suspicious relapse on surveillance, and patients with low positive TMs after chemotherapy; and high risk (90\% to 100\% risk), which included patients with gross clinical metastatic germ cell tumors before starting chemotherapy, patients with testicular mass before orchiectomy, patients with CS IS, and patients with obvious viable (high positive TMS) residual disease after chemotherapy.

two independent experiments conducted in the same laboratory and in two different laboratories was observed (Pearson $r=0.97$ and 0.90, respectively; Data Supplement).

\section{Whole-Disease Results, Characteristics, Clinical Events, and miR371 Expression Analysis Grouped by Risk of Harboring aGCM}

Individual clinical profiles were aggregated to create clinically logical risk and tumor volume clusters for harboring aGCM to demonstrate the performance of miR371 analytics within low-, moderate-, and high-risk clusters. Risk groups were defined by stringent clinical criteria with known predictive characteristics as follows and as detailed in the Data Supplement.

Low-risk designation was assigned to individuals with risk estimates based on the composite clinical picture (imaging, classic marker elevation, and pathologic features of primary tumor) of less than $25 \%$ chance of harboring or developing aGCM. The low-risk cluster had aGCM tumor volumes

TABLE 1. Two-by-Two Table of GCM Cases and Controls by miR371 Expression or No miR371 Expression

miR371 Expression $\begin{gathered}\text { GCT History With Clinically } \\ \text { Confirmed aGCM (No. of cases) }\end{gathered}$

Plasma miR371 expr

Plasma miR371 expression on prospectively obtained sample

No plasma miR371 expression on prospectively obtained sample
GCT History With No

Clinically Confirmed GCM (to March 2019; No. of controls)
Total No.

44 positive tests

44 TPS O FPs 44 positive tests

86 TNs

88 negative tests

Total samples 46 with confirmed aGCM 86 with no confirmed aGCM 132

NOTE. Sensitivity: 44 TPs/44 TPs + 2 FNs = 44/46 (96\%). Specificity: 86 TNs/86 TNs + 0 FPs = 86/86 (100\%). Positive predictive value = 44 TPs/44 TPs + O FPs $=44 / 44$ (100\%). Negative predictive value $=86 \mathrm{TNs} / 86 \mathrm{TNs}+2 \mathrm{FNs}=86 / 88$ (98\%) (to June 15, 2019). Median follow-up time from prospectively obtained sample was 15 months (range, 4 to 36 months). Median follow-up time from time of risk designation was 19 months (range, 5 to 187 months). Abbreviations: aGCM, active germ cell malignancy; FN, false negative; FP, false positive; GCT, germ cell tumor; TN, true negative; TP, true positive. 


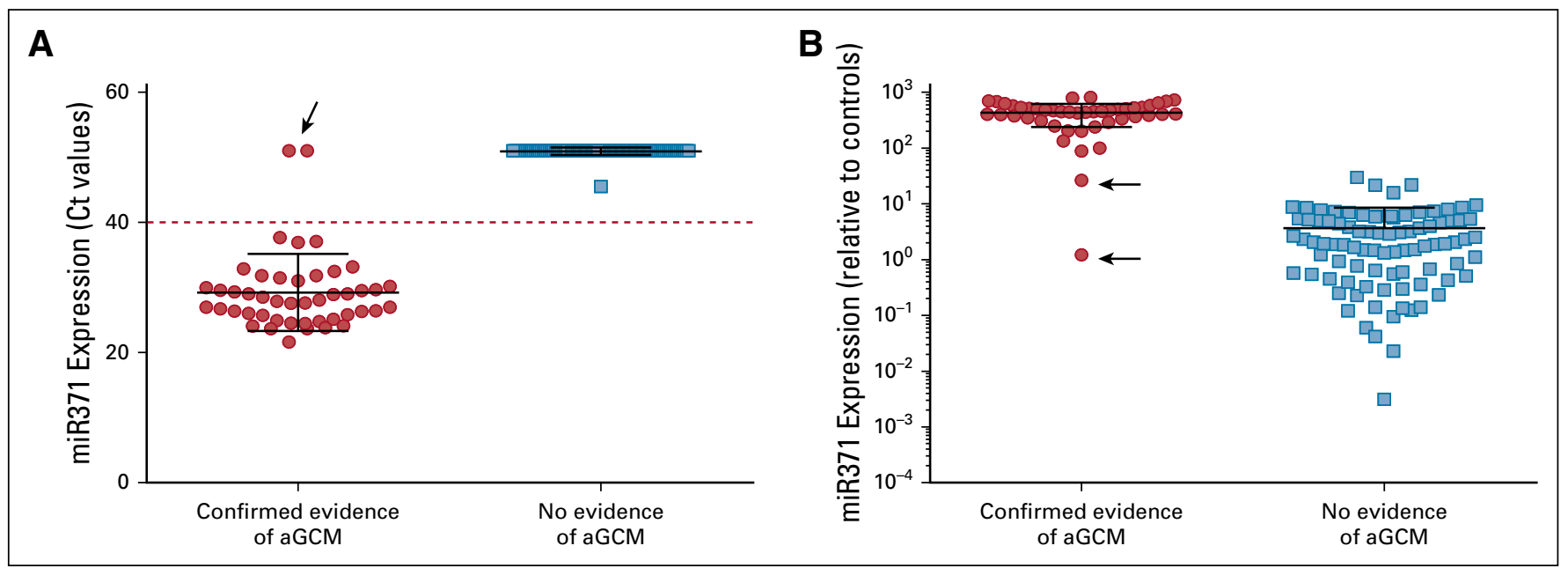

FIG 3. miR371 is expressed in patients with clear evidence of active germ cell malignancy. Plasma miR371 was evaluated in the 132 specimens divided as cases and controls, as reported in Table 1. (A) miR371 cycle threshold (Ct) raw values were used to qualitatively assess miR371 expression, using a cutoff value of $40 \mathrm{Ct}$ (dashed line). (B) miR371 expression was quantified as fold of expression increase relative to the negative controls, after normalization for miR30b-5p, miR39-3p, and miR-451. The black arrows indicate the two patients with false-negative results. aGCM, active germ cell malignancy.

ranging from no malignancy to subclinical volumes of germ cell malignancy (GCM; $<1 \mathrm{~cm}$ with normal markers). ${ }^{16,17}$ Moderate-risk designation was assigned to individuals whose composite clinical picture predicted a $25 \%$ to $50 \%$ chance of harboring or developing aGCM and who, as a group, had GCM volumes from none to microscopic or subclinical (clinical stage IB nonseminoma) ${ }^{18,19}$ to lower end of clinical detectability (1- to 2-cm nodal enlargement and/or mildly abnormal classic markers) and in whom there was a $20 \%$ to $30 \%$ likelihood of FP clinical findings. ${ }^{20}$ Highrisk designation was assigned based on a composite picture highly suggestive or certain of aGCM and consisted primarily of patients with GCT with definitive pathologic, imaging, and/or serologic evidence of aGCM and a risk estimate for harboring aGCM exceeding $90 \%$. There is no clinical presentation that can predict a risk of harboring GCM in the 50\% to $90 \%$ range. Twenty-one individuals in the high-risk group were rendered, by virtue of treatment, to low- or moderate-risk status and were subject to a second confirmation (or not) of aGCM. These patients had postorchiectomy or postchemotherapy samples drawn for miR371 analysis.

Table 2 lists the detailed clinical characteristics by risk and volume cluster and results of miR371 expression analysis of prospectively obtained samples by risk cluster and in total. The low-risk, low-volume cluster $(n=56)$ had an unexpectedly low rate of developing confirmed aGCM (one of 56 patients; $2 \% v 5 \%$ to $25 \%$ predicted). ${ }^{21}$ This low rate of recurrence may be related to the follow-up time of less than 3 years or, more likely, to the strict stipulations of absolutely normal classic markers and strict imaging criteria ( $<1-\mathrm{cm}$ axial diameter adenopathy). The single patient in the low-risk cluster expressing miR371 is the only patient who developed aGCM (Data Supplement).
Specificity and PPV of miR371 expression for aGCM in the low-risk cluster were $100 \%$ and $100 \%$, respectively. Median follow-up from study entry for the low-risk group was 14.5 months (range, 4 to 35 months). With the limitation of this follow-up, no FNs were identified, and the sensitivity and NPV were $100 \%$ and $100 \%$, respectively (Data Supplement).

In the high-risk cluster $(n=35)$ with a predicted risk of aGCM exceeding 90\%, 32 (91\%) of 35 patients had aGCM confirmed, and 32 of 32 patients with confirmed aGCM expressed miR371. Two (6\%) of 35 patients in the high-risk group, despite high expert suspicion for aGCM, had clinical confirmation of conditions that were not aGCM (two TNs), and neither patient had detectable miR371 expression (Fig 2). One (3\%) of 35 patients had pathologically confirmed aGCM but negative miR371 expression (FN; Data Supplement). For the high-risk group, the specificity and PPV of miR371 was 100\% and $100 \%$, respectively, and the sensitivity and NPV were $97 \%$ and $67 \%$, respectively (Data Supplement).

The moderate-risk group $(n=41)$, with a predicted risk of aGCM of $25 \%$ to $50 \%$, was the most informative. Overall, 12 (29\%) of 41 patients demonstrated clinically confirmed aGCM. The suspected median tumor volume and median marker elevation were, by design, at or less than the lower limits of detectability and reliability for classic clinical determinants. All patients lacked definitive marker elevation or bulky imaging findings, consistent with the risk cluster definitions. Within the moderate-risk group, miR371 expression was demonstrated in all but one patient with clinically confirmed aGCM (11 of 12 patients; 92\%). One patient had clinically confirmed aGCM by pathology but did not have miR371 expression demonstrated in plasma 
TABLE 2. Clinical Risk Clusters and miR371 Expression

No./Total No.

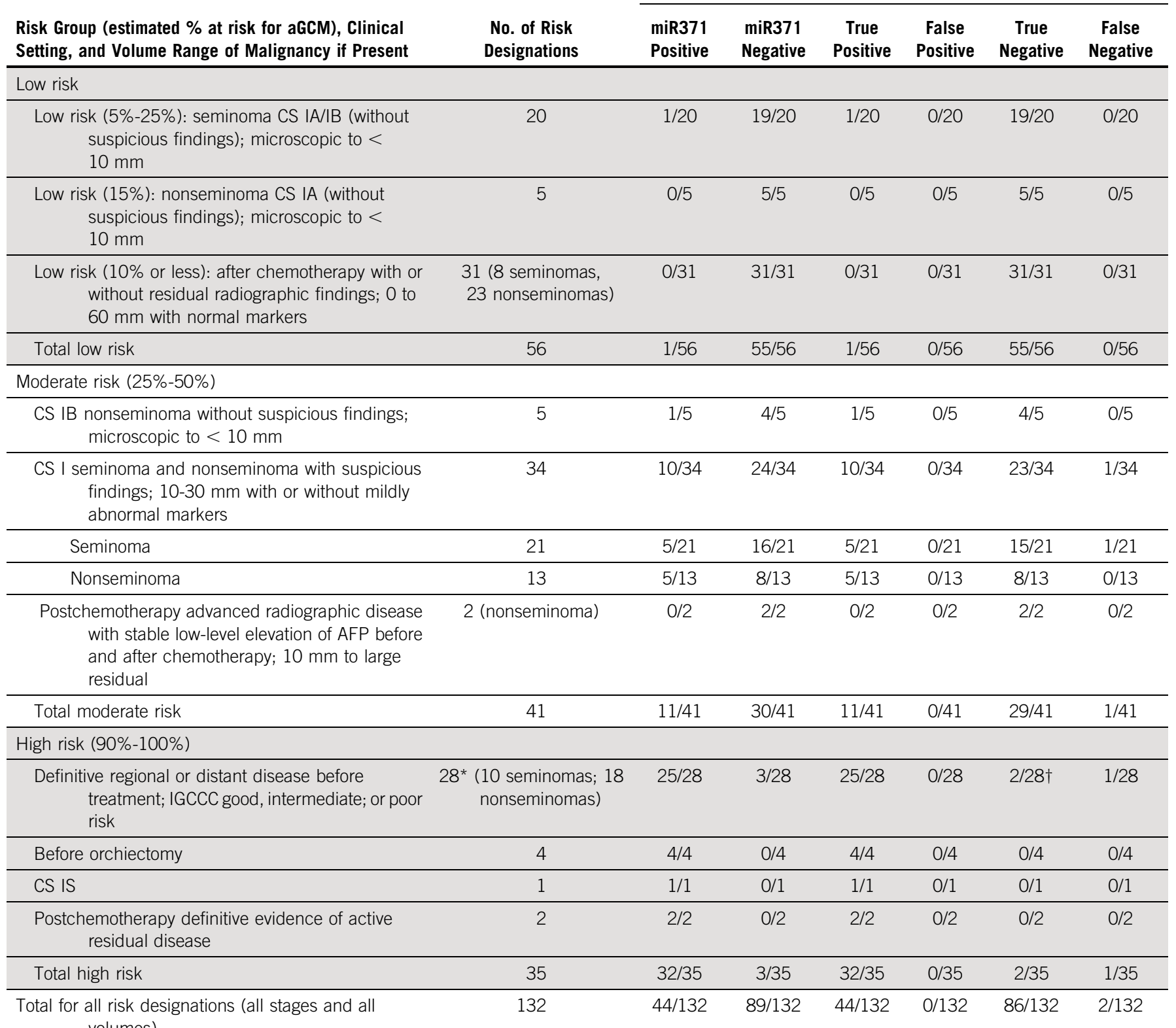
volumes)

Abbreviations: AFP, $\alpha$-fetoprotein; aGCM, active germ cell malignancy; CS, clinical stage; IGCCCG, International Germ Cell Cancer Collaborative Group.

*IGCCCG risk classification: good, $n=24$ (seminoma, $n=9$; nonseminoma, $n=15$ ); intermediate, $n=2$ (seminoma, $n=1$; nonseminoma, $n=1$ ); and poor, $\mathrm{n}=2$.

†No evidence of viable germ cell tumor on pathology (fibrosis and teratoma and rhabdomyosarcoma).

samples obtained before the clinical confirmation (one FN; Data Supplement). miR371 specificity and PPV for aGCM in the moderate-risk group were $100 \%$ and $100 \%$, respectively. Sensitivity and NPV in this group (to date) were $92 \%$ and $97 \%$, respectively. Median follow-up times for the moderate-risk group from diagnosis and from prospective collection were 20 months (range, 8 to 97 months) and 15 months (range, 5 to 31 months), respectively (Data Supplement).

\section{Operating Characteristics of miR371 Compared With Tumor Markers and CT Imaging in Detecting Nonteratoma Viable GCM}

A secondary outcome of our trial design was the ability to compare miR371 expression analysis with concurrent individual classic clinical determinants (ie, CT, $\beta$-HCG, AFP, and $L D H$ ) with receiver operating characteristic curves of miR371 in the whole cohort and in the moderaterisk group (Fig 4). In the whole cohort, the miR371 area 
A

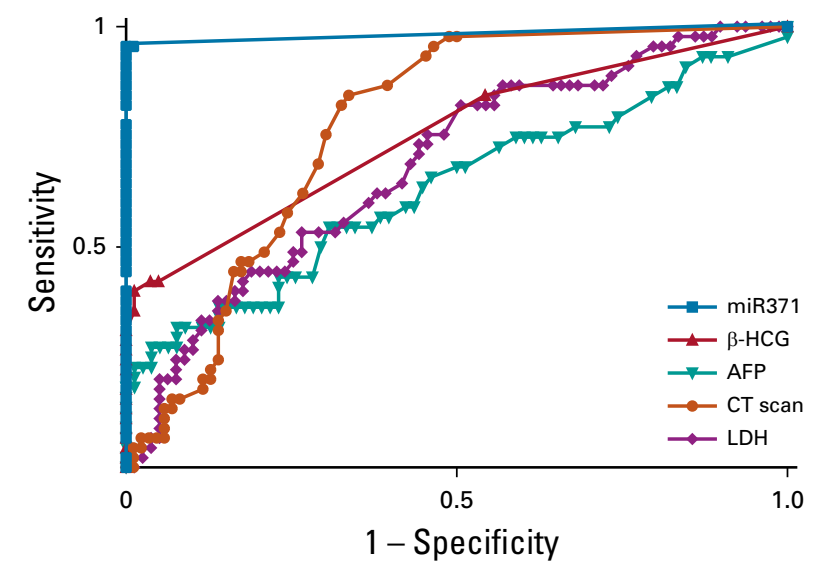

B

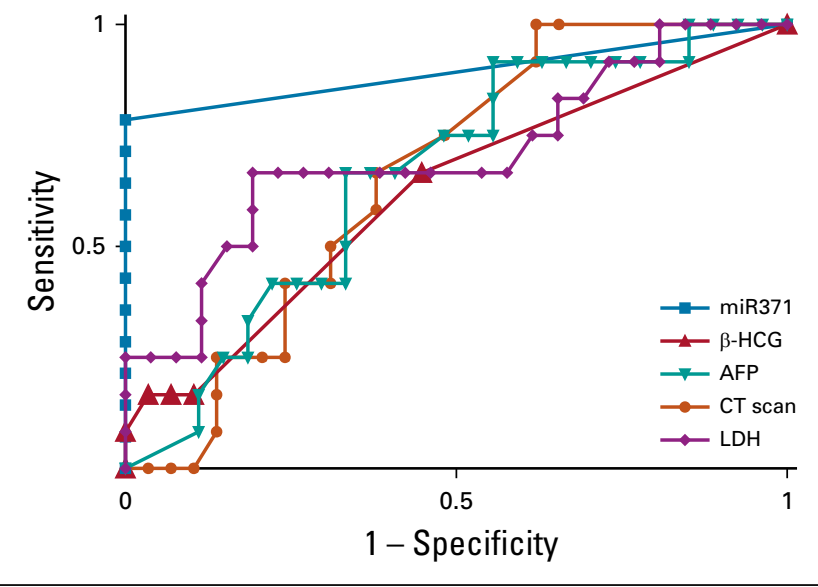

FIG 4. Areas under the receiver operating characteristic curve of miR371, computed tomography (CT) scan, and the classic germ cell tumor markers $\alpha$-fetoprotein (AFP), $\beta$-human chorionic gonadotropin ( $\beta$-HCG), and lactate dehydrogenase (LDH) of (A) the whole cohort of samples $(n=132)$ and $(B)$ samples from the moderate-risk group. The patients were classified as cases or controls, as reported in Table 1.

under the curve was $0.97(95 \% \mathrm{Cl}, 0.9434$ to 1.013$)$ compared with $0.77(95 \% \mathrm{Cl}, 0.6845$ to $0.8639 ; P<.001)$ for CT scan and 0.63, 0.75, and 0.68 for AFP, $\beta$-HCG, and $\mathrm{LDH}$, respectively (Data Supplement). In addition, in the moderate-risk group, in which uncertainty is a significant clinical challenge, 22,23 miR371 outperformed the gold standard diagnostic methods, as illustrated in the Data Supplement.

\section{DISCUSSION}

Our exploratory study was designed to sufficiently define operating characteristics of miR371 expression in aGCM using a prospective, inductive, whole-disease and longitudinal approach to underpin the development of large, definitive, real-world studies to fully define the practical clinical utility of miR371 expression in GCT. Our primary conclusion is that we have confirmed that miR371 expression, analyzed using low-cost, plasma-based methodology and with qualitative reporting (expressed or not expressed), has high specificity and PPV (100\% and $100 \%$, respectively, in our study) for the presence of aGCM. It is also noteworthy that miR371 expression outperformed classic markers and imaging at the lowest levels of current clinical detection and reliability (moderate-risk group).

The sensitivity and NPV of miR371 expression in aGCM are incompletely defined by our prospective pilot trial, and the information available from older retrospective trials does not complete the sensitivity and NPV picture. In the clinical setting, sensitivity and NPV are operating characteristics that can only be fully determined after sufficient follow-up.

In March 2019, a multi-institutional biospecimen collection and clinical data aggregation effort with centralized serumbased analysis of miR371 expression was reported. ${ }^{14}$ The primary focus of the article by Dieckmann et al ${ }^{14}$ was in the prediagnostic setting before orchiectomy, and their study used classic noncancer controls. Pilot trials by English and Dutch groups demonstrated that miR371 could be identified reliably in patients who had intact suspected aGCM down to a tumor size of $1 \mathrm{~cm}$, and this central prediagnostic question was confirmed and further informed by Dieckmann et al. ${ }^{14}$ Other conclusions of the effort are limited by the lack of follow-up and the limited clinical data available for more advanced patients. However, there were some general trends that could be identified, including the fact that miR371 declines rapidly with effective chemotherapy and an apparent association between tumor bulk and higher quantitative levels of miR371.

The primary clinical utilities and practice-changing outcomes of the upcoming large studies of miR371 are mostly dependent on its high specificity and high PPV. If the PPV results seen in our pilot trial hold up across the spectrum of local and small-volume regional disease in larger trials, a meaningful consequence should be reduction in less accurate, more expensive, radiation-associated, and bothersome body imaging, which is the current backbone of active and post-treatment surveillance. The accuracy of miR371 in identifying small-volume aGCM predicted from our study would also suggest a role in postchemotherapy assessment for aGCM of residual masses (which is currently based on surgical resection or frequent imaging). ${ }^{24,25}$ In our study, 36 patients had postchemotherapy samples obtained (34 TNs and two TPs), which yielded 100\% specificity, PPV, and NPV for aGCM with a follow-up time after chemotherapy exceeding 15.5 months (range, 3 to 35 months). Although the sensitivity and NPV await full definition in the postorchiectomy clinical stage I setting, miR371 expression in the postorchiectomy setting or in the 
setting of active surveillance before developing classic clinical abnormalities likely represents persistent or progressive microscopic disease after orchiectomy. The presence of miR371 expression in the immediate postorchiectomy period could drive rational assignment of adjuvant treatments (a single cycle of bleomycin, etoposide, and cisplatin or primary retroperitoneal lymph node dissection). In addition, miR371 expression at the time of clinical presentation or relapse with marker-negative stage IIA disease can improve the accuracy of the clinical diagnosis of recurrent GCM and render unnecessary the biopsies or continued serial observation currently needed to proceed confidently to treatment.

Our primary end point was not to perform a prospective stand-alone study of miR371 in GCT, but to move this concept forward with alacrity, if appropriate. Moving to a large practical study to refine the full operating characteristics of miR371 expression analysis in the real-world setting is warranted by strong signals of clinical utility. Spurred by the early recognition in our pilot trial of high

\section{AFFILIATIONS}

${ }^{1}$ BC Cancer, Vancouver Centre, University of British Columbia, Vancouver, British Columbia, Canada

${ }^{2}$ Vancouver Prostate Centre, University of British Columbia, Vancouver, British Columbia, Canada

${ }^{3}$ BC Cancer, Vancouver, British Columbia, Canada

${ }^{4}$ Huntsman Cancer Institute, University of Utah, Salt Lake City, UT

${ }^{5}$ University of South California, Los Angeles, CA

${ }^{6}$ Princess Margaret Cancer Centre, Toronto, Ontario, Canada

${ }^{7}$ Testicular Cancer Commons, Beaverton, OR

${ }^{8}$ SWOG Group Chairs Office, Portland, OR

${ }^{9}$ Faculty of Medicine, University of Coimbra, Coimbra, Portugal

\section{CORRESPONDING AUTHOR}

Christian Kollmannsberger, MD, Division of Medical Oncology, BC Cancer, Vancouver Cancer Centre, University of British Columbia, 600 W 10th Ave, Vancouver, British Columbia, Canada V5Z 4E6; e-mail: ckollmannsberger@bccancer.bc.ca.

\section{EQUAL CONTRIBUTION}

C.N. and C.K. contributed equally as senior coauthors to this work.

\section{PRIOR PRESENTATION}

Presented in part at the ASCO 2018 Genitourinary Cancers Symposium, San Francisco, CA, February 8-10, 2018; the 54th Annual Meeting of the American Society of Clinical Oncology, Chicago, IL, June 1-5, 2018; and the European Society for Medical Oncology 2018 Congress, Munich, Germany, October 19-23, 2018.

\section{SUPPORT}

Supported by the Genito-Urinary Medical Oncology Canada-Astellas Research Grant 2017 No. $20 R 10950$ (C.K. and L.N.) and in part by National Institutes of Health/National Cancer Institute Grants No. U10CA180888 (C.D. Blanke, primary investigator) and U10CA180819 (M. Leblanc, primary investigator) for pilot work for SWOG trial S1823. specificity with no FPs and the early recognition of three informative discordant cases, the move to large-scale, realworld testing has been accomplished with the accelerated development and recent approval of the concept by the National Cancer Institute of S1823, the SWOG/National Clinical Trials Network North American study that will answer the clinical utility questions around miR371 expression in early-stage GCT. S1823 will coordinate clinical data and biospecimens exchange with Children's Oncology Group AGCT 1523 trial (Active Surveillance, Bleomycin, Carboplatin, Etoposide, or Cisplatin in Treating Pediatric and Adult Patients With Germ Cell Tumors; ClinicalTrials.gov identifier: NCT03067181), which addresses the clinical utility of miRNAs in more advanced GCT. S1823 and Children's Oncology Group AGCT 1523 should provide information sufficient to refine the Cls for sensitivity and specificity, NPV, and PPV of microRNA expression analysis across the spectrum of GCT and, most importantly, identify a number of areas of clinical utility that can potentially change practice in GCT management and reduce patient burden.

Also supported by the SWOG Young Investigator Training Course and SWOG Translational Science Course (L.N.). The content is solely the responsibility of the authors and does not necessarily represent the official views of the National Institutes of Health.

\section{AUTHORS' DISCLOSURES OF POTENTIAL CONFLICTS OF INTEREST} AND DATA AVAILABILITY STATEMENT

Disclosures provided by the authors and data availability statement (if applicable) are available with this article at DOI https://doi.org/10.1200/ JCO.18.02057

\section{AUTHOR CONTRIBUTIONS}

Conception and design: Lucia Nappi, David Huntsman, Brock O'Neil, Martin Gleave, Craig Nichols, Christian Kollmannsberger

Financial support: Christian Kollmannsberger

Administrative support: Craig Nichols, Christian Kollmannsberger Provision of study materials or patients: Lucia Nappi, Bernie J. Eigl, Kim Chi, Jean Michel Lavoie, Craig Nichols, Christian Kollmannsberger Collection and assembly of data: Lucia Nappi, Marisa Thi, Amy Lum, Bernie J. Eigl, Christopher Martin, Benjamin L. Maughan, Kim Chi, Peter C. Black, Martin Gleave, Jean Michel Lavoie, Daniel Khalaf, Craig Nichols, Christian Kollmannsberger

Data analysis and interpretation: Lucia Nappi, David Huntsman, Bernie J. Eigl, Christopher Martin, Alan So, Peter C. Black, Martin Gleave, Alex W. Wyatt, Robert Bell, Siamak Daneshmand, Robert J. Hamilton, Ricardo R.N. Leao, Craig Nichols, Christian Kollmannsberger

Manuscript writing: All authors

Final approval of manuscript: All authors

Accountable for all aspects of the work: All authors

\section{ACKNOWLEDGMENT}

We thank the valued input from Michael LeBlanc, PhD, SWOG Biostatistics; James Rae, PhD, SWOG Translational Science Executive Officer; Lindsay Frazier, MD, Children's Oncology Group study chair; and Matthew Murray, PhD, Cambridge, United Kingdom. 


\section{REFERENCES}

1. Einhorn LH: Treatment of testicular cancer: A new and improved model. J Clin Oncol 8:1777-1781, 1990

2. Eini R, Dorssers LC, Looijenga LH: Role of stem cell proteins and microRNAs in embryogenesis and germ cell cancer. Int J Dev Biol 57:319-332, 2013

3. Gillis AJ, Stoop HJ, Hersmus R, et al: High-throughput microRNAome analysis in human germ cell tumours. J Pathol 213:319-328, 2007

4. Palmer RD, Murray MJ, Saini HK, et al: Malignant germ cell tumors display common microRNA profiles resulting in global changes in expression of messenger RNA targets. Cancer Res 70:2911-2923, 2010

5. Voorhoeve PM, le Sage C, Schrier M, et al: A genetic screen implicates miRNA-372 and miRNA-373 as oncogenes in testicular germ cell tumors. Cell 124 : 1169-1181, 2006

6. Gillis AJ, Rijlaarsdam MA, Eini R, et al: Targeted serum miRNA (TSmiR) test for diagnosis and follow-up of (testicular) germ cell cancer patients: A proof of principle. Mol Oncol 7:1083-1092, 2013

7. Murray MJ, Halsall DJ, Hook CE, et al: Identification of microRNAs from the miR-371 373 and miR-302 clusters as potential serum biomarkers of malignant germ cell tumors. Am J Clin Pathol 135:119-125, 2011

8. Murray MJ, Raby KL, Saini HK, et al: Solid tumors of childhood display specific serum microRNA profiles. Cancer Epidemiol Biomarkers Prev 24:350-360, 2015

9. Dieckmann KP, Radtke A, Spiekermann M, et al: Serum levels of microRNA miR-371a-3p: A sensitive and specific new biomarker for germ cell tumours. Eur Urol 71:213-220, 2017

10. Looijenga LH, Gillis AJ, Stoop H, et al: Relevance of microRNAs in normal and malignant development, including human testicular germ cell tumours. Int J Androl 30:304-315, 2007

11. Murray MJ, Huddart RA, Coleman N: The present and future of serum diagnostic tests for testicular germ cell tumours. Nat Rev Urol 13:715-725, 2016

12. Mitchell PS, Parkin RK, Kroh EM, et al: Circulating microRNAs as stable blood-based markers for cancer detection. Proc Natl Acad Sci USA 105:10513-10518, 2008

13. Spiekermann M, Belge G, Winter N, et al: MicroRNA miR-371a-3p in serum of patients with germ cell tumours: Evaluations for establishing a serum biomarker. Andrology 3:78-84, 2015

14. Dieckmann KP, Radtke A, Geczi L, et al: Serum levels of microRNA-371a-3p (M371 test) as a new biomarker of testicular germ cell tumors: Results of a prospective multicentric study. J Clin Oncol 37:1412-1423, 2019

15. Radtke A, Hennig F, Ikogho R, et al: The novel biomarker of germ cell tumours, micro-RNA-371a-3p, has a very rapid decay in patients with clinical stage 1. Urol Int 100:470-475, 2018

16. Chung P, Daugaard G, Tyldesley S, et al: Evaluation of a prognostic model for risk of relapse in stage I seminoma surveillance. Cancer Med 4:155-160, 2015

17. Steyerberg EW, Keizer HJ, Fosså SD, et al: Prediction of residual retroperitoneal mass histology after chemotherapy for metastatic nonseminomatous germ cell tumor: Multivariate analysis of individual patient data from six study groups. J Clin Oncol 13:1177-1187, 1995

18. Albers $\mathrm{P}$, Siener R, Kliesch S, et al: Risk factors for relapse in clinical stage I nonseminomatous testicular germ cell tumors: Results of the German Testicular Cancer Study Group trial. J Clin Oncol 21:1505-1512, 2003

19. Nicolai N, Miceli R, Necchi A, et al: Retroperitoneal lymph node dissection with no adjuvant chemotherapy in clinical stage I nonseminomatous germ cell tumours: Long-term outcome and analysis of risk factors of recurrence. Eur Urol 58:912-918, 2010

20. Stephenson AJ, Bosl GJ, Bajorin DF, et al: Retroperitoneal lymph node dissection in patients with low stage testicular cancer with embryonal carcinoma predominance and/or lymphovascular invasion. J Urol 174:557-560, 2005

21. Kollmannsberger C, Tandstad T, Bedard PL, et al: Patterns of relapse in patients with clinical stage I testicular cancer managed with active surveillance. J Clin Oncol 33:51-57, 2015

22. Hamilton RJ, Nayan M, Anson-Cartwright L, et al: Treatment of relapse of clinical stage I nonseminomatous germ cell tumors on surveillance. J Clin Oncol 37 : 1919-1926, 2019

23. Kollmannsberger CK, Nappi L, Nichols C: Management of stage II germ cell tumors: Be sure, be patient, be safe. J Clin Oncol 37:1856-1862, 2019

24. Leão R, van Agthoven T, Figueiredo A, et al: Serum miRNA predicts viable disease after chemotherapy in patients with testicular nonseminoma germ cell tumor. J Urol 200:126-135, 2018

25. Daneshmand S, Albers P, Fosså SD, et al: Contemporary management of postchemotherapy testis cancer. Eur Urol 62:867-876, 2012 


\section{AUTHORS' DISCLOSURES OF POTENTIAL CONFLICTS OF INTEREST}

\section{Developing a Highly Specific Biomarker for Germ Cell Malignancies: Plasma miR371 Expression Across the Germ Cell Malignancy Spectrum}

The following represents disclosure information provided by authors of this manuscript. All relationships are considered compensated unless otherwise noted. Relationships are self-held unless noted. I = Immediate Family Member, Inst = My Institution. Relationships may not relate to the subject matter of this manuscript. For more information about ASCO's conflict of interest policy, please refer to www.asco.org/rwc or ascopubs.org/jco/site/ifc.

Open Payments is a public database containing information reported by companies about payments made to US-licensed physicians (Open Payments).

\section{Lucia Nappi}

Honoraria: Astellas Pharma, Pfizer

Patents, Royalties, Other Intellectual Property: Ivermectin analogue compound therapeutics for heat shock protein-27 (HSP27) inhibition, methods and uses associated therewith provisional No.: 62/756,707

Travel, Accommodations, Expenses: Pfizer

David Huntsman

Employment: Contextual Genomics

Leadership: Contextual Genomics

Stock and Other Ownership Interests: Contextual Genomics

Bernie J. Eigl

Honoraria: Pfizer, Janssen, AstraZeneca, Merck, Roche Canada

Consulting or Advisory Role: Roche Canada, AstraZeneca, Merck, Janssen

Speakers' Bureau: Merck

Travel, Accommodations, Expenses: Janssen, AstraZeneca

Benjamin L. Maughan

Consulting or Advisory Role: Janssen Oncology, Exelixis, Tempus, Peloton

Travel, Accommodations, Expenses: Exelixis

Kim Chi

Honoraria: Sanofi, Janssen, Astellas Pharma, Bayer

Consulting or Advisory Role: ESSA, Astellas Pharma, Janssen, Sanofi, Amgen, Bayer, AstraZeneca, Roche

Research Funding: Janssen (Inst), Astellas Pharma (Inst), Bayer (Inst), Sanof (Inst), Tokai Pharmaceuticals (Inst), Eli Lilly/ImClone (Inst), Bristol-Myers Squibb (Inst), Merck (Inst), Roche (Inst)

\section{Alan So}

Honoraria: Janssen, Amgen, Bayer, Tersera, Astellas Pharma

Speakers' Bureau: Janssen

Patents, Royalties, Other Intellectual Property: Patent: Sitka

Expert Testimony: Janssen

Peter C. Black

Consulting or Advisory Role: Abbvie, Astellas Pharma, Janssen Oncology, Amgen, Bayer, Merck, Sanofi Canada, Biosyent, Ferring, Roche Canada, MDxHealth, AstraZeneca, Urogen Pharma, Asieris, Bristol-Myers Squibb Speakers' Bureau: TerSera

Research Funding: iProgen, GenomeDx, Sitka

Patents, Royalties, Other Intellectual Property: PCT/CA2014/000787, Canada, 2014-11-03, Cancer Biomarkers and Classifiers and uses thereof; \#61899648 United States, 2013-03-13, Bladder cancer signature

Travel, Accommodations, Expenses: Sanofi

\section{Martin Gleave}

Stock and Other Ownership Interests: OncoGenex

Honoraria: Janssen, Astellas Pharma, Bayer, Abbvie, Sanofi, Amgen,

MDxHealth, GDx

Consulting or Advisory Role: Janssen, Astellas Pharma, Bayer, Abbvie, Sanofi, Amgen, MDxHealth, AstraZeneca, GDx

Research Funding: Janssen, Astellas Pharma, Bayer

Patents, Royalties, Other Intellectual Property: OncoGenex: OGX-011, OGX427

\section{Alex W. Wyatt}

Consulting or Advisory Role: Genzyme

Speakers' Bureau: Janssen

Research Funding: Janssen

Jean Michel Lavoie

Honoraria: Pfizer, Astellas Pharma

Daniel Khalaf

Consulting or Advisory Role: Bayer

\section{Siamak Daneshmand}

Honoraria: Photocure, QED, Olympus, MDxHealth, Ferring, Spectrum Pharmaceuticals, Pacific Edge

Consulting or Advisory Role: Photocure, Taris, Ferring, QED

Research Funding: Photocure

Travel, Accommodations, Expenses: Photocure

Robert J. Hamilton

Honoraria: Janssen, Abbvie, Bayer, Astellas Pharma, Amgen

Research Funding: Janssen, Bayer

Travel, Accommodations, Expenses: Janssen

Ricardo R.N. Leao

Travel, Accommodations, Expenses: Roche Pharma AG

Christian Kollmannsberger

Honoraria: Pfizer, Novartis, Bristol-Myers Squibb

Consulting or Advisory Role: Pfizer, Novartis, Bristol-Myers Squibb, Astellas

Pharma, Ipsen, Eisai, Janssen

Travel, Accommodations, Expenses: Pfizer, Novartis, Eisai

No other potential conflicts of interest were reported. 